\title{
Anaesthesia for the Elderly Patient (Second Edition)
}

\author{
Chris Dodds, Chandra M. Kumar, Frederique Servin. Oxford Anaesthesia Library; 2017, \\ 162 pages. ISBN: 9780198735571
}

\author{
Heather McDonald, MD, FRCPC
}

Received: 11 May 2017/Accepted: 26 May 2017/Published online: 5 June 2017

(C) Canadian Anesthesiologists' Society 2017

The stated intent of this book is to "present current information on the care of the elderly patient, in an accessible and easy to understand format." Certainly, the book is a comprehensive resource, well organized into short chapters, and presented in a consistent style.

The first chapter focuses on the epidemiology and economic impact of the aging population. Authors Chris Dodds, Chandra M. Kumar, and Frederique Servin provide an accurate, insightful summary of the current state of providing anesthetic care for the elderly population. They also make the compelling argument that, as our elderly population is increasing, the need to provide care to them in a way that maintains functionality and independence is becoming an increasingly important social, financial, and medical concern.

The next few chapters focus on the pathophysiology of aging and the relevant pharmacologic changes that occur. Of particular use is the authors' attempt to distinguish between the expected changes associated with normal aging compared with the changes that result from common comorbid illnesses in the elderly population. I expect these chapters will be particularly excellent references for most medical students despite the presumed specificity for anesthesia practitioners.

The chapter on "Pre-operative Assessment and Preparation of Elderly Patients Undergoing Major Surgery" advocates thorough risk stratification and optimization by a multidisciplinary team prior to major elective surgery. Although this overview is excellent and

H. McDonald, MD, FRCPC ( $\bowtie)$

Department of Anesthesia, University of Manitoba, Winnipeg, MB, Canada

e-mail: hmcdonald101@yahoo.ca the spectrum of potential decline in function is thoroughly addressed, it is rather too general to be of any real assistance in the management of individual patients. Specific assessment tools and relevant investigations would be more appropriately determined from existing published national guidelines and other resources dedicated to perioperative assessment. The authors also make the interesting point that, although the oldest patients are at increased surgical risk and prolonged recovery, neither is suggested to be a barrier to elective surgery. The case is even made, in a subsequent chapter, that day-case anesthesia may be appropriate, even advantageous, in carefully selected elderly patients.

Emergency and elective surgical procedures are handled separately in this book, with orthopedic, abdominal, neurosurgical, urologic, and gynecologic interventions addressed independently. These chapters identify, to varying degrees, common considerations, conflicts, and suggested intraoperative management strategies. Postoperative care and suggestions for the management of anesthesia are covered in an additional chapter. Because there is so much disparity in the postoperative course and analgesic needs, this information may have been more useful if arranged according to specific surgical interventions. Including pain and the postoperative course as considerations in each of the surgery-specific chapters might have improved the specificity and individualization of recommendations. Of note, the highest-risk surgical procedures, such as thoracic, vascular, and cardiac surgery, are not addressed.

The chapter on the provision of intensive care for the elderly patient provides interesting insight on, and discussion of, the concepts of functional capacity and organ reserve, noting their importance as disease processes 
when determining intensive care unit outcomes. The authors argue for careful preadmission evaluation and thorough discussions with the patient and family regarding expectations and potential outcomes. Although the point is made that determining functional limitations and acceptable outcomes for older patients is particularly challenging, the suggestions are sound medical practice for more than just the elderly population. The same is true for the new chapter in this edition of the book titled, "Anaesthesia for Non-Theatre Environments." This chapter comprehensively reviews the challenges of providing anesthesia at remote sites. Although accurate and insightful, the information is in no way specific to the elderly patient. A similar argument applies to the equally well-written final chapter on ethics, which is an excellent overview and applies to all patients from whom physicians seek consent. Cognitive dysfunction and sleep disorders are the only specific postoperative complications that are addressed in devoted chapters - an appropriate distinction considering the scope and magnitude of these problems in the elderly.

Each chapter includes a useful initial overview in the form of a "Key Points" section. The initial and concluding chapters have a more general "Summary" section as well, which is unfortunately missing from the chapters devoted to specific surgical interventions. Although there is also a helpful "Further Reading" list, the chapters themselves are poorly referenced. In contrast, the reprinted tables and graphs included throughout have a readily identified reference and are simple and easily understood. They certainly add to the accessibility and reference value of this publication.

Overall, the second edition of Anaesthesia for the Elderly Patient is a well-organized, comprehensive review of anesthesia for the elderly patient. Some sections, such as the detailed discussion of the physiology of aging, could serve as an excellent, complete resource. Other areas, such as preoperative evaluation and postoperative pain management, are good overviews, but supplemental reading is required. Although the book is obviously aimed at anesthesia providers caring for elderly patients, there are many sections, particularly in the concluding chapters, entirely appropriate for younger patients and health-care professionals treating patients of all ages.

Conflicts of interest None declared.

Editorial responsibility This submission was handled by Dr. Hilary P. Grocott, Editor-in-Chief, Canadian Journal of Anesthesia. 